\title{
Etiology, diagnosis, and clinical management of vulvodynia
}

This article was published in the following Dove Press journal:

International Journal of Women's Health

2 May 2014

Number of times this article has been viewed

\author{
Leslie A Sadownik \\ University of British Columbia, \\ Department of Obstetrics and \\ Gynecology, Vancouver, BC, Canada
}

Correspondence: Leslie A Sadownik Sixth Floor, Department of Gynecology, Gordon and Leslie Diamond Health Care Center, 2775 Laurel Street, Vancouver, BC, Canada V5Z IM9

$\mathrm{Tel}+\mathrm{I} 6048754260$

Fax + I 6048754869

Email leslie.sadownik@vch.ca

\begin{abstract}
Chronic vulvar pain or discomfort for which no obvious etiology can be found, ie, vulvodynia, can affect up to $16 \%$ of women. It may affect girls and women across all age groups and ethnicities. Vulvodynia is a significant burden to society, the health care system, the affected woman, and her intimate partner. The etiology is multifactorial and may involve local injury or inflammation, and peripheral and or central sensitization of the nervous system. An approach to the diagnosis and management of a woman presenting with chronic vulvar pain should address the biological, psychological, and social/interpersonal factors that contribute to her illness. The gynecologist has a key role in excluding other causes for vulvar pain, screening for psychosexual and pelvic floor dysfunction, and collaborating with other health care providers to manage a woman's pain. An important component of treatment is patient education regarding the pathogenesis of the pain and the negative impact of experiencing pain on a woman's overall quality of life. An individualized, holistic, and often multidisciplinary approach is needed to effectively manage the woman's pain and pain-related distress.
\end{abstract}

Keywords: vulvodynia, diagnosis, treatment, etiology, sexual pain disorder, dyspareunia, vestibulodynia, assessment, treatment, multidisciplinary

\section{Introduction}

The International Society for the Study of Vulvar Diseases defines vulvodynia as chronic pain or discomfort involving the vulva for more than 3 months and for which no obvious etiology can be found. ${ }^{1}$ If the etiology is evident (eg, lichen sclerosus), then the woman has chronic vulvar pain secondary to lichen sclerosus, not vulvodynia. Thus, vulvodynia is a diagnosis of exclusion. In fact, women with vulvodynia often do not use the word "pain" to describe their discomfort. Rather they use phrases such as itching, burning, stinging, irritation, stabbing, and/or rawness. The classification of vulvodynia is currently based on a description of the pain. A woman's symptoms may be "generalized" to the whole vulva (generalized vulvodynia) or "localized" to a specific area such as the clitoris (clitorodynia) or the vestibule of the vagina (vestibulodynia). The pain may be "provoked" (caused by direct touch, inserting a tampon, or sexual touch), "unprovoked" (present without touch), or have a "mixed" pattern. ${ }^{1}$

The characteristics of a woman's pain may wax and wane over time. Thus, the classification of vulvodynia is not exclusive and there is often overlap between the categories. ${ }^{2}$ A woman may initially notice pain at the vaginal opening during sexual contact ("it only hurts when I have intercourse"), then begin to notice monthly episodes of unprovoked vestibular discomfort ("it feels like I get a yeast infection every month before my period"), and finally, daily episodes of unprovoked vulvar discomfort ("I feel burning 
all over the vulva all the time"). Thus, the same woman may be "diagnosed" with provoked vestibulodynia (PVD) or generalized vulvodynia depending on when she seeks help from a clinician. Over the years, descriptive terms have evolved. For example, the terms "dysesthetic vulvodynia", "essential vulvodynia", and "burning vulvar syndrome" have all been used in the past in lieu of the current term "generalized vulvodynia". Given the confusion about terms, it is important that the clinician understands that these terms are descriptors and not distinct disease entities. The term vulvodynia, however, is helpful in validating to women that chronic vulvar pain can occur in the absence of any obvious etiology and that it is a recognized legitimate medical condition.

Vulvodynia affects women of all ages, reproductive stages, and ethnicities. ${ }^{3,4}$ The lifetime prevalence of this condition has been estimated at $8 \%$, and this prevalence remains constant across all decades up to the age of 70 years. ${ }^{4}$ The bulk of the literature regarding vulvodynia focuses on PVD because this appears to be the most common clinical presentation. PVD is also thought to be the most common cause of sexual pain in women under the age of 30 years $^{5}$ and typically affects women $20-40$ years of age.

Vulvodynia is a significant burden to society, the health care system, the affected woman, her family, and/or her intimate partner. It has been estimated that the annual economic burden of vulvodynia in the US is $\$ 31-\$ 72$ billion. ${ }^{6}$ This cost includes direct health care costs, indirect health care costs (eg, transportation to hospital), and indirect societal costs (eg, sick leave). Secondly, vulvodynia is a significant burden to the health care system. Women with vulvodynia may seek help from multiple health care providers, including family doctors, gynecologists, dermatologists, urologists, and alternative health practitioners. These clinicians may not be familiar with the symptoms and/or signs of vulvodynia. This results in multiple visits to health care providers, and ultimately a delay in diagnosis and management. Even when the clinician recognizes the symptoms as being compatible with vulvodynia, he or she may be stymied as to how to manage the woman's pain. Standard evidence-based practice guidelines do not exist for the management of vulvodynia. Thus, caring for women with vulvodynia is often perceived as a real challenge. Not surprisingly, the health care provided to women with vulvodynia is often not perceived as helpful by the women. ${ }^{7}$ It is important to note that many women "suffer in silence" and do not seek medical help. In one telephone survey, less than half of women who met screening criteria for vulvodynia reported seeking medical help. ${ }^{4}$ Thirdly, vulvodynia has a significant negative impact on a woman's psychosexual health ${ }^{8}$ and overall quality of life. ${ }^{9}$ The chronicity of vulvar pain may result in women experiencing frustration, ${ }^{8}$ anxiety, ${ }^{8}$ chronic stress ${ }^{10}$ and depression. ${ }^{11}$ Many women with vulvodynia experience pain with sexual activity and the majority report sexual difficulties. Women with PVD report diminished sexual self-esteem, satisfaction, interest, and arousal (decreased lubrication and inability to achieve orgasm) ${ }^{7,12}$ They also report a restricted repertoire of sexual behavior. ${ }^{13}$ Women with PVD often refuse a partner's sexual advances and avoid sexual contact in an effort to reduce or avoid pain. ${ }^{14}$ They describe having to plan and/or mentally prepare themselves for sex. ${ }^{14}$ Considerably less research has focused on experiences of lesbian women with PVD, although my clinical experience is that they appear to be affected in the same ways as heterosexual women.

The chronic nature of the pain and the woman's response to that pain has an adverse effect on her interpersonal relationships. For example, a woman may stay at home because of severe daily pain and thus become socially isolated. If the woman is unable to work and/or contribute to household activities, this may place a burden on other members of her household. Sexual partners witness and react to a woman's experience of sexual pain. This may affect their own sexual health, and their reactions may also facilitate or diminish the woman's pain. ${ }^{15}$ Over half of couples dealing with sexual pain secondary to PVD reported viewing themselves as different from other "sexually healthy couples" and needing to make special accommodations to the woman's vulvar pain. ${ }^{16}$

The gynecologist in general practice plays a pivotal role in helping woman with vulvodynia. The purpose of this paper is to provide guidance for the clinician who may be unfamiliar with the diagnosis and/or the principles of how a biopsychosocial model can be applied to the effective treatment of women with vulvodynia. At the beginning of my clinical practice, my management of women with vulvodynia focused on medical and surgical interventions to address the biological pain. After 15 years of practice exclusively in the area of vulvovaginal diseases, those interventions have been replaced by other therapies that my experience and evidence supports to be equally if not more effective. This paper introduces the clinician to psychologic and behavioral therapies that may be integrated into his or her own practice and/or provided in collaboration with other health care professionals to help reduce a woman's pain and pain-related distress secondary to vulvodynia.

\section{Etiology}

Women with vulvodynia are often very distressed about not being able to identify a "cause" for their pain. The etiology 
of vulvodynia is uncertain and likely multifactorial. There is debate about whether the pain is primarily caused by a local insult or injury, or a maladaptive peripheral and/or maladaptive central pain processing mechanism. At a local level, there may be an initial trigger that causes inflammation and/or injury affecting the vulva. This results in repetitive stimulation of pain receptors and ultimately receptor or nerve damage (nociceptive pain). ${ }^{17}$ Allergic contact dermatitis ${ }^{18}$ and yeast ${ }^{19,20}$ or other urogenital infections ${ }^{21}$ have all been proposed as causes for inflammation. Women may identify trauma as precipitating their pain. Direct injury to their vulva (eg, episiotomy) or direct (eg, forceps) and indirect injury (eg, back or hip injury) to the pelvic floor may be recalled as preceding the pain. Other women report the onset of pain to be associated with a hormonal trigger (eg, starting the birth control pill, postpartum amenorrhea, or perimenopause). Chronic local inflammation may result in an increase in: neurotransmitters and immune-mediated factors; proliferation of unmyelinated nerve fibers; chronic stimulation of afferent primary c fibers; and chronic stimulation of the dorsal horn cells in the spinal cord. Histologic evidence of local changes to the vestibular skin in women with PVD includes chronic inflammatory infiltration, including increased mast cells and plasma cells, and increased nerve fibers. ${ }^{22,23}$ Thus, the acute nociceptive pain may result first in peripheral sensitization and ultimately central sensitization, ie, neuropathic pain. This sensitization prolongs the symptoms long after the original tissue injury.

Conversely, the initial trigger may not involve peripheral sensitization of the vulva, but rather begin with generalized urogenital, ${ }^{24,25}$ pelvic, and/or central nervous sensitivity. Women with vulvodynia have been found to have bladder sensitivity, urinary urgency, and frequency. ${ }^{24}$ Further, women with complaints of urinary burning have been found to be at increased risk for developing vulvodynia. ${ }^{25}$ Pelvic sensitivity may be secondary to injury and/or inflammation of the pelvic organs and/or musculoskeletal components of the pelvis. Women with secondary PVD may report an antecedent history of chronic pelvic pain and/or deep dyspareunia. Women with vulvodynia have been found to have generalized hypersensitivity, ${ }^{26}$ a decreased pain threshold in the vulvar area $^{27}$ as well as other areas of the body, ${ }^{27-29}$ and enhanced pain processing. ${ }^{26}$

Central sensitization may in fact precede the peripheral sensitization. It may be triggered by a noxious stimulus or an innocuous stimulus. For example, it has been proposed that some people are more likely to develop chronic pain after an injury because of their reaction to the pain. The "fear avoidance model of pain" suggests that people can react to pain by either confronting (adaptive response) or avoiding (maladaptive response) the pain. Those who avoid it, demonstrate pain-related fear, pain-related anxiety, and hypervigilance of perceived impending pain, leading to avoidance behavior. ${ }^{30}$ Women with PVD demonstrate many of these maladaptive emotional, cognitive, and behavioral reactions to their pain. They catastrophize about their pain, ie, think about the pain (ruminate), exaggerate the pain (magnification of pain), and fear their pain. ${ }^{31}$ While these reactions to the experience of pain are understandable, they may have a key role in developing chronic pain after an injury. As previously noted, a partner's solicitous responses (eg, expressing sympathy and comfort) or negative responses (eg, expressing anger or hurt) to a woman's sexual pain may reinforce her maladaptive response to sexual pain. ${ }^{15}$

A noxious input to the central nervous system may not be necessary if the system is sufficiently primed or sensitized. Basson $^{32}$ proposed that if the system is sufficiently sensitized (eg, by chronic stress), it may be activated by a low-threshold innocuous stimulus. Thus, central sensitization may not only be responsible for acute pain becoming chronic, but may in fact be the cause of the original pain. Women with sexual pain secondary to PVD have been found to have psychologic vulnerabilities that may lead to chronic psychologic distress. Women with a prior history of anxiety and/or depression were found to be four times more likely to develop PVD. ${ }^{33}$ Women with PVD have also been described as having anxiety-like predispositions, being perfectionists, and fearful of negative evaluation. ${ }^{34}$ While some of these reactions may result from chronic vulvar pain, in other cases these vulnerabilities were present before the onset of pain, and may have altered key central pain processing mechanisms. It is important to note that there does not appear to be a reported increase in the prevalence of emotional, physical, or sexual abuse in this group when compared with the population at large. ${ }^{35}$

These etiologic theories position vulvodynia as a neuropathic disorder. However, some authors have suggested that vulvodynia is a somatoform ${ }^{36}$ or functional pain disorder, ie, a type of somatic pain syndrome similar to fibromyalgia, interstitial cystitis, and/or irritable bowel syndrome. A functional pain disorder is one where the patient experiences chronic pain ( $>3$ months) for which there is no known cause or any visible physical injury or disease, and the pain causes clinically significant distress or impairment. The association of vulvodynia with other common chronic pelvic pain conditions like interstitial cystitis, irritable bowel syndrome, and chronic pelvic pain may support this hypothesis. Forty-five 
percent of women with vulvodynia report having at least one of the following chronic pain conditions: chronic fatigue syndrome, endometriosis, fibromyalgia, interstitial cystitis, or irritable bowel syndrome. ${ }^{37}$

It is often impossible for a woman with vulvodynia and/or her clinician to confidently identify a single biological "cause" for her pain. Successful treatment of a woman's vulvodynia goes beyond identifying a single trigger for the pain, prescribing a single medical treatment, and/or following a generic "vulvodynia" algorithm. The chronicity of the pain predictably gives rise to significant problems concerning a woman's psychological, sexual, and pelvic floor health. It may be unclear whether these comorbidities are causative, associative, or as a result of the vulvodynia. However, the diagnosis and treatment of women with vulvodynia involves recognition and assessment of all the factors influencing her experience of pain. The focus of treatment should be on the woman in pain, and not the pain in isolation.

\section{Clinical diagnosis}

Assessment and effective management of a woman with vulvodynia needs to be individualized. Given the confusion regarding the terminology related to vulvodynia, this section discusses management of the woman's symptoms, ie, daily chronic vulvar pain and/or sexual pain. The gynecologist should inform the patient at the beginning of the first visit that it will require more than one appointment to complete her assessment and develop an appropriate management plan. Table 1 highlights the different aspects of patient assessment and counseling that may be spread over several visits.

\section{Patient interview}

The goals of the first clinical interview are: to gather relevant information about the patient's pain and her medical and psychosexual history; validate that the patient's pain is real; provide education and support; and provide recommendations regarding skin care and symptom management. A detailed pain history should be obtained from the patient (Table 2). Providing a pain diary for patients to complete can help identify factors that influence their pain. Physicians need to consider a broad differential diagnosis when assessing vulvar complaints (Table 3). A thorough medical, surgical history, and systems review is necessary to exclude another clinical diagnosis (eg, recurrent yeast vaginitis, endometriosis) that may be contributing to her chronic vulvar symptoms.

At the first visit, basic screening questions should be asked regarding a woman's psychosexual history. In particular, the woman should be asked what the impact of her
Table I Goals for assessment and initial management of vulvodynia

\begin{tabular}{llll}
\hline Focus of encounter & Visit I & Visit 2 & Visit 3 \\
\hline Clinical history: in-depth pain history & $\times$ & & \\
Examination to exclude other causes & $\times$ & & \\
Patient education: diagnosis & $\times$ & & \\
Recommend: skin care, symptom control & $\times$ & & \\
Follow-up discussion: review pain diary & & $\times$ & \\
In-depth sexual history & $\times$ & \\
Patient education: psychosexual "fall out" & $\times$ & \\
Counseling: sexual & $\times$ & \\
Repeat examination: assess pelvic floor & & & $\times$ \\
Teach pelvic floor exercises & & $\times$ \\
Individualize treatment recommendations & & \\
\hline
\end{tabular}

pain has been on her life. How has her sex life changed? More detailed information regarding her past psychosexual history may be deferred until subsequent visits when rapport is established. Many women have been made to feel that the "pain is all in their heads" and thus may be defensive about this line of questioning and withhold information. However, screening questions may identify factors that could be contributing to her pain and pain-related distress, such as a history of abuse, current relationship problems, and ongoing mood disorders. In these situations, a referral to an appropriate health care provider (eg, sexual therapist, psychologist, relationship counselor) for further assessment and care may be indicated.

\section{Key symptoms from the history}

Women with moderate to severe daily chronic pain are often postmenopausal and report constant burning or aching pain that affects the entire vulva and may even radiate onto the thighs, buttocks, and up into the abdomen. Classically, nothing seems to provoke the pain, "it is just there all of the time", ie, generalized unprovoked vulvodynia. The pain may interfere with wearing clothing, walking, sitting for

Table 2 Pain history

\begin{tabular}{lll}
\hline P & Precipitates & What caused the pain? \\
$\mathrm{P}$ & Provokes & What provokes the pain? \\
$\mathrm{P}$ & Palliates & What palliates the pain? \\
$\mathrm{Q}$ & Quality & What is the quality of the pain? \\
$\mathrm{R}$ & Radiation & Does the pain radiate? Where? \\
$\mathrm{S}$ & Severity & What is the severity? \\
& & What activities are limited due to pain? \\
& & (daily, sexual) \\
$\mathrm{T}$ & Temporal & How has the pain changed over time? \\
& & Have there been any pain-free intervals of time? \\
$\mathrm{T}$ & Treatment tried & What treatments have been tried to date? \\
& & Patient compliance? \\
\hline
\end{tabular}


Table 3 Causes of vulvovaginal symptoms and dyspareunia

\begin{tabular}{ll}
\hline Etiology & Examples \\
\hline Infectious & Vulvitis: herpes, tinea, candidiasis \\
& Vaginitis: candidiasis, trichomoniasis, atrophic \\
& Cervicitis: chlamydia, gonorrhea \\
& Pelvic inflammatory disease \\
& Acute cystitis \\
& Vulvar dermatitis: irritant, contact \\
Inflammatory & Vulvar dermatoses: lichen sclerosus, psoriasis \\
& Vulvovaginitis: atrophic, endometriosis, \\
& Adenomyosis, interstitial cystitis, \\
inflammatory bowel disease \\
Traumatic & Vulvar fissures \\
Functional & Imperforate hymenal ring, vaginal septum \\
Musculoskeletal & Inhibited sexual arousal \\
\hline
\end{tabular}

long periods, and sleep. Typically, sexual activity does not significantly aggravate this constant pain. The patient's goal of treatment is usually relief of the daily vulvar pain. Women who suffer from daily vulvar discomfort may be unable to participate in activities of daily living. In severe cases, they may become housebound and unable to carry out household chores, work, and/or socialize. ${ }^{?}$

In contrast, women reporting vestibular sensitivity that results in sexual pain are usually younger. They often describe feeling like the vagina is too small when penetration is attempted, that there is something "blocking" the vagina, or "tearing" of the skin at the perineum after intercourse. These symptoms and signs may be suggestive of associated hypertonicity of the pelvic floor muscles. After intercourse they report distressing vestibular burning and external dysuria that may last for hours. Typically, if the woman avoids inserting anything into her vagina, she is pain-free. Thus, the goal of treatment is usually the relief of the distressing sexual pain. As the degree of the localized sensitivity of the vestibule increases, women may notice external dysuria not associated with penetration, intermittent flare-up of vulvar pain (often mistaken as "monthly yeast infections"), and daily burning at the vaginal opening.

Key symptoms that suggest an alternative diagnosis include a history of vulvar itch that the patient reports as scratching (dermatitis), abnormal vaginal bleeding and/or discharge (lichen planus), pain with sitting and bowel movements, or other neurologic symptoms (eg, pudendal neuralgia). A history of atopy and/or other skin conditions also suggests a dermatologic cause for the discomfort. A detailed review of the patient's vulvar hygiene regimen is necessary to identify behaviors, irritants, and/or allergens that may contribute to her discomfort. A history of preceding chronic pelvic pain, dysmenorrhea, or deep dyspareunia may suggest that the superficial dyspareunia is secondary to an underlying primary cause (eg, endometriosis).

\section{Clinical examination}

Women with vulvodynia may devote all of their health care visits to finding pain relief and thus neglect routine health examinations and screening tests. Incorporating general screening tests such as blood pressure measurement and a Papanicolaou smear into the examination should be considered if overdue. The specific goals of the clinical examination for women with vulvodynia include exclusion of other diagnoses, patient education, and localization of her vulvar pain. It is worth noting that many women with vulvodynia have had negative experiences with gynecologic examinations, and may be very anxious and even fearful about the upcoming clinical examination. Deferring the examination to a subsequent visit may be necessary.

It is helpful to have the patient observe the clinical examination of the vulva, and this may be facilitated with a handheld mirror. The gynecologist should begin by reviewing the anatomy of the vulva. The vulva and perianal area should be inspected methodically, (Table 4), beginning at one point (mons), systematically examining all surfaces, and noting changes in skin texture and color. If there are significant objective changes to the appearance of the skin, in particular changes to the texture of the skin (eg, erosions, plaques) a biopsy should be considered to rule out vulvar intraepithelial neoplasia. The histologic diagnosis of an inflammatory skin condition (eg, dermatoses) will only be reliable if the patient has been off topical steroids for at least 2 weeks. Next, inspect the anatomy. Changes to the vulvar anatomy (loss of the labia minora, burying of the glans of the clitoris) are suggestive of lichen sclerosus and/or lichen planus. Examine the area for inflammation and/or lesions.

Next, the patient should be asked to describe the location of her pain and quantify the pain prior to palpation of the vulva. A visual pain analog scale (rated 0-10) is a helpful tool to help the patient quantify their pain. The vulva should be palpated with light consistent pressure using a moistened cotton swab in a clockwise manner (mons, labia majora, and perineal and perianal skin areas). The patient is asked to report if the area that is being palpated is painful and if the palpation provokes and/or makes the pain worse. The labia minora should be gently separated by the clinician or the patient herself, and the vestibule inspected. Gently pulling the labia up, rather than apart, allows for easier viewing of the vestibule. If possible, a moistened cotton swab should be used to lightly palpate 
Table 4 Systematic approach to examining the vulva

\begin{tabular}{lll}
\hline & Steps & Questions \\
\hline S & Skin & Is the skin normal? (texture, color) \\
A & Anatomy & Is the anatomy/architecture of the vulva normal? \\
I & Inflammation & Is there any inflammation? (margins, color, degree) \\
L & Lesions & Are there any lesions? Use dermatological terms \\
& & to describe \\
\hline
\end{tabular}

the vestibule (Skene's glands, minor vestibular glands, posterior fourchette) and identify any painful areas. The vestibule is normally a sensitive area, and reports of mild sensitivity (1-3/10) may be within the normal range of sensitivities. Women with PVD may report hyperalgesia (increased sensitivity to a painful stimulus) and allodynia (pain from a nonpainful stimulus) during the examination. However, they will have normal bulbocavernosus and anal reflexes. Due to the innervation of the vagina (autonomic and pudendal nerves), pain is often poorly localized. Women with sexual pain secondary to PVD often think the "problem" is "inside" the vagina and involves a large area. They are surprised to visualize that the painful area is confined to the vestibule, located only at the entrance to the vagina, and is a small area. This observation will help them to feel more able to control their pain.

If the vestibule is sensitive, lidocaine $2 \%$ can be applied topically. The vestibule can be palpated again 3 minutes later to see if the sensitivity has decreased enough to allow for an internal examination. If possible, a narrow speculum should be inserted into the vagina. The vaginal mucosa should be inspected for signs of atrophy, inflammation, and/or adhesions. The speculum should then be removed and palpation of the rest of the lower genital tract performed.

A single digit can be inserted just beyond the hymenal ring into the vagina in order to assess the tone and function of the pelvic floor muscles to judge the depth of the levator ani muscle bulk from the perineal body and the relative size of the muscular opening between these muscles from right to left. ${ }^{38}$ The greater the tone, the further superior the muscle opening is from the perineal body and the smaller and tighter the opening. A high tone will often result in the clinician having to insert the examining finger "up and over" these muscles to enter into the vagina. The woman should then be asked to contract her pelvic floor muscles to assess her ability to control the muscles, the strength of the contraction, and ability to relax the muscles fully after the contraction. Patients with pelvic floor dysfunction will often demonstrate poor contractile function, slow and hesitant release of the contraction, and release back to a tense pelvic floor resting tone.
The vaginal walls, deep pelvic floor muscles, ischial spines, and base of the bladder should be sequentially palpated. Changes to the vaginal anatomy such as adhesions and/or constriction rings are signs of an erosive vaginitis (eg, lichen planus). Palpation of the ischial spines will provoke pain in women with pudendal neuralgia. Tenderness under the urethra or bladder neck may signal interstitial cystitis. Women with pelvic floor dysfunction may also demonstrate tenderness along the obturator internus muscles and elevated fascial tension (arcus tendineus fascia pelvis and/or arcus tendineus levator ani). ${ }^{38}$ Finally, a bimanual examination of the uterus, cul de sac, and adnexa should be performed to rule out pelvic pathology that may give rise to deep dyspareunia.

If the patient cannot tolerate an internal examination because of pain or pelvic floor tightness, the examination should be deferred until there is diminished vulvar pain, although not indefinitely. Patients with lifelong pelvic floor tightness may have anatomical defects, such as a vaginal septum. Likewise, women with erosive lichen planus may present with minimal vestibular findings but have inflammation, erosions, and/or scarring in the vagina, suggesting an erosive vaginitis.

\section{Laboratory investigations}

Patients with vulvodynia are often misdiagnosed as having an infectious vaginitis. The vaginal discharge can be assessed by testing the $\mathrm{pH}$ and obtaining a vaginal sample that can be sent for a Gram stain and culture. A Gram stain of the vaginal discharge will identify the presence (or absence) of lactobacilli, clue cells, white blood cells, and active yeast elements. An acidic pH, presence of lactobacilli, and absence of clue cells will exclude bacterial vaginosis. A negative vaginal culture may be helpful in ruling out yeast vaginitis, whereas a positive culture may not differentiate between colonization and infection. A review of all past vaginal Gram stains and cultures will help to assess the likelihood of recurrent yeast vaginitis.

\section{Key findings of vulvodynia}

Typically women with primarily daily chronic unprovoked pain (generalized vulvodynia) report the presence of moderate burning prior to palpation, and palpation may not necessarily provoke further pain. In contrast, women with sexual pain (PVD) will be pain-free prior to palpation and then report significant pain with palpation of the vestibule. The key criteria for diagnosing PVD are a history of severe pain during attempted vaginal entry and tenderness to pressure (palpation with a cotton swab) localized to the vestibule in the absence 
of other pathology. The presence of focal vestibular erythema (around the minor vestibular glands and/or Bartholin's ducts) is a common but inconsistent finding with PVD, and not an indication for skin biopsy. It is important to note that the presence of vestibular tenderness and diffuse moderate erythema can be found in other inflammatory conditions, such as lichen planus, plasma cell vulvitis, and graft versus host disease. A biopsy may not necessarily be helpful in diagnosis of these conditions. Gynecologists need to be aware of the limitations of a skin biopsy and the subsequent pathologist's report. The biopsy is a sample of the area, and depending on the location, depth, and size, it may not necessarily be representative of the underlying vulvar disease. Vulvar disease experts often rely on discriminating facts from the patient's history and their clinical examination to recognize patterns of disease. The pathologic findings often support the working diagnosis while not necessarily being diagnostic. Thus, if there is any question about a possible underlying skin condition, then a referral to an expert in vulvar diseases is strongly recommended. In most cases, the clinician will find minimal objective findings when assessing women with vulvodynia. In the absence of other clinical findings, further laboratory investigations and/or imaging studies (eg, ultrasound or computed tomography scan) are not indicated. A thorough clinical examination is essential to reassure the patient that other diagnoses have been systematically excluded.

\section{Clinical management}

The first step in helping the woman and couple is to validate that the pain is "real" and that it has a name, ie, "vulvodynia". The woman and/or her partner may have at some point wondered if it was "all in her head". It is very important that the partner and/or support person hears directly from the clinician that vulvodynia is a legitimate pain condition. Information that is relevant to discuss includes: the prevalence of this condition, possible causes for the original acute pain, how acute pain can become chronic, and the negative impact of pain on psychologic health (eg, depression) and sexual health (eg, loss of sexual desire). It is helpful to use a pelvic model and/or clear anatomic diagrams to reinforce what areas of the vulva are affected and the relationship between the vulva, pelvic floor muscles, peripheral nerves, and central nervous system. The clinician should introduce the concept that the pain experience is multidimensional and that other factors are likely contributing to her pain. The woman and her partner should be given ample opportunity for questions. After the woman and her partner understand the diagnosis, they can then be referred to other resources for more information (books, website, and patient associations). Women and their partners in our treatment program appreciate getting information directly from the physician, as they have found other sources of information (eg, Internet) very confusing.

The second step is to provide suggestions regarding skin care and symptom management. Treatment recommendations should be patient-centered, not diseasecentered. Many management algorithms for the treatment of vulvodynia do not take into consideration the patient's characteristics and the context in which she is experiencing pain. Women should be encouraged to shift their focus from finding out exactly "what" precipitated the discomfort to managing the discomfort. Promotion of self-management strategies in individuals with chronic pain may be associated with increased self-efficacy, improved internal locus of control for pain, and reduced health care visits. ${ }^{39}$ An important step in managing this discomfort is to stop all inappropriate treatments. A review of their past and current treatments often reveals a long list of ineffective therapies, such as topical antiyeast, antibacterial, steroid, and hormonal creams.

Further, women often attempt to "wash their symptoms away" by using chemicals (eg, antibacterial soaps) and physical practices (eg, frequent bathing) that are damaging to the skin. The clinician should review these and provide the patient with a handout outlining good skin care and vulvar hygiene practices. Daily use of a hypoallergenic moisturizer or barrier cream applied to the vulva should be recommended to help hydrate and protect the skin.

Mild sexual discomfort may be reduced with topical anesthetics, eg, lidocaine $2 \%-5 \%$, prior to penetration. Topical lidocaine may cause a contact dermatitis, and patients should be cautioned to discontinue use if they develop a reaction to the medication. The couple should only attempt penetrative sex when the woman is in a state of high sexual arousal because the physical changes associated with arousal will help make intercourse more comfortable. The woman and her partner should be encouraged to explore strategies to reduce her discomfort (use of lubricants, different sexual positions, using a reverse Kegel's prior to insertion). Women who are experiencing moderate or severe pain with sex should be advised to avoid the painful sexual activity. Avoidance of intercourse will not cause the pain to go away but it will reduce the association between sex and pain.

Recommending local estrogen replacement in perimenopausal and postmenopausal women with vulvodynia is the first step in addressing vulvar discomfort in this age group. 
Local estrogen therapy has been found to ameliorate the negative impact of vulvovaginal atrophy on the sexual health of postmenopausal women, ${ }^{40}$ and the combination of estrogen and local lidocaine effectively treats severe unprovoked postmenopausal vestibulodynia. ${ }^{41}$ It is my opinion that adequate estrogen replacement therapy in this age group requires both intravaginal therapy and topical estrogen therapy (compounded in a hypoallergenic base) to the vestibule. The role of topical estrogen in treating reproductive-aged women who are well estrogenized is unknown.

Patient adherence to recommendations may be affected by the stress of the initial visit, receiving too much information, and/or receiving information that disagrees with her beliefs. Thus, a critical goal of the first visit is to provide a diagnosis she understands and accepts, while confidently excluding other diagnoses that she may secretly fear (eg, cancer, sexually transmitted infections). In-depth discussion of treatment options may be best deferred until the patient has a chance to process the relevant information about her diagnosis. For example, if a patient is convinced her pain is due to a yeast infection, she will expect the gynecologist to provide antifungal therapy. If instead she receives a diagnosis of vulvodynia (something she has never heard of before) and is given a prescription for an antidepressant, in all likelihood she will not fill that prescription. She may not even return to the doctor. At our center, $34.7 \%$ of women presenting for assessment to our multidisciplinary vulvodynia program report choosing not to follow an earlier doctor's treatment recommendation.

Patients who have been suffering for 3-6 months may respond to reassurance regarding the benign nature of the condition, simple skin care, and pain relief recommendations. While the natural history of vulvodynia is unknown, spontaneous remission rates of up to $56 \%$ have been reported. ${ }^{42}$ However, patients who have been experiencing pain for more than 6 months, in particular women with sexual pain, will benefit from a more holistic approach and additional counseling. Follow-up visits will need to assess further the other factors that are likely contributing to her ongoing pain, ie, psychosexual concerns and pelvic floor muscle dysfunction, and provide further pain management (Figure 1).

\section{Psychologic and sexual therapy}

How women with vulvodynia think and feel about their pain has an impact on their pain experience. Supportive psychotherapy and cognitive behavior therapy (CBT) are both effective for women experiencing sexual pain secondary to PVD. ${ }^{43}$ As previously noted, many women with PVD have negative self-perception ("I'm not normal") and thought patterns ("I will never have pain-free sex") that can influence their experience of pain. A gynecologist can reduce a woman's distress by explaining the relationship between negative thoughts ("I am not normal"), feelings (eg, shame), and behaviors (eg, not socializing). It is helpful for the gynecologist to help patients identify and challenge these problematic thoughts. "Yes I have vulvodynia. Other women have vulvodynia and many have got better. One day my pain will go away."

Education alone has been found to be helpful in reducing psychologic and sexual distress in women with sexual pain due to PVD. ${ }^{44}$ Thus, while a gynecologist may not feel comfortable about providing in-depth sexual counseling, he or she can relieve a woman's sexual distress by providing basic sexual education. First, one can begin by discussing a woman's normal sexual response cycle and how pain can have a predictable negative impact on this cycle. This sexual "fall out" can be framed as an expected consequence of experiencing pain during sex. The decreasing frequency and breadth of sexual intimacy within a relationship is often a result of the woman's attempt to avoid further pain, and her feelings of shame and/or perceived "failure" associated with attempts at sexual intercourse. Male partners often worry that their female partner is "not interested in them" or that they are "doing something wrong". On the other hand, a woman's partner may be the one to withdraw sexually because he or she may be very anxious about causing pain to the partner. The woman may interpret this withdrawal as rejection and further "proof" that she is sexually "inadequate". These unspoken beliefs and behaviors can lead to tension in the relationship and a breakdown in communication. Having a health professional frame this sexual fall out as an understandable and predictable outcome of experiencing sexual pain will help

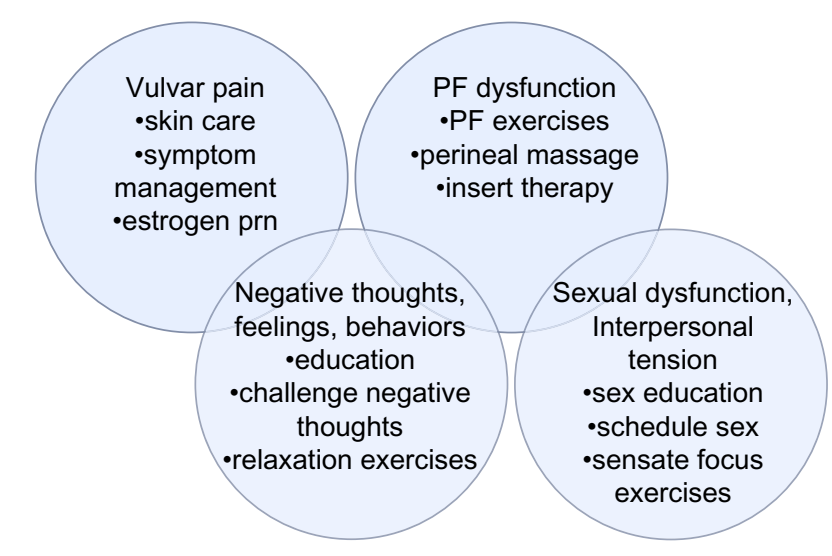

Figure I Biopsychosocial framework for treating women with vulvodynia. Abbreviations: PF, pelvic floor; prn, as needed. 
couples to stop blaming themselves and each other, and begin to develop skills to move forward.

Many couples have got out of the "habit" of being sexual, and often describe their relationship as one of "platonic roommates". Scheduling and/or planning time for sex will help the woman to prepare for it and feel more in control. Couples should be explicit with each other about what sexual activities they will and will not engage in. Painful sex needs to be taken off the "sexual menu". Many couples have limited "sexual scripts" and may not easily be able to replace intercourse with other forms of sexual intimacy. A list of resources (videos, books) may be helpful for these couples.

If the relationship is stable (ie, with emotional intimacy), then couples should be encouraged to work first on their physical intimacy and not always link physical touch (hugging, kissing) with sexual foreplay. Women often avoid or are aversive to any physical touch because they are afraid it will signal to their partner that they are willing to be sexual. Couples should then be encouraged to engage in pleasurable nonpenetrative sensual and/or sexual activity. Some couples may need to start slowly with sensate focus exercises. This will allow the woman to let down her guard, relax, tune into her body, and enjoy the physical pleasure associated with touch. Automatic negative thoughts may emerge outside of and during sexual activity. For example, the expectation of pain with sexual intercourse ("I know this will hurt, it always hurts") will result in ambivalence about having intercourse, reduced arousal/lubrication, and involuntary muscle contraction with penetration, leading to further pain. The cycle becomes self-perpetuating, with every painful sexual experience reinforcing the woman's avoidance of sex or limiting her sexual experiences to brief painful attempts at intercourse. Staying in the moment ("right now we are just kissing and I enjoy kissing"), rather than anticipating pain and/or judging her own sexual performance is crucial to breaking this cycle.

Couples may find it difficult to communicate about and address interpersonal and sex-related problems. They may feel awkward about scheduling sex, engaging in sensate focus activities, or expanding their sexual repertoire. Lovemaking is a skill that benefits from deliberate practice. If both partners "cannot find the time" to talk about their problems or be sexual (avoidance behaviors), seeking the services of a sexual health professional can be crucial for improvement.

\section{Physical therapy}

Chronic daily vulvar and sexual pain can be associated with changes to the pelvic floor muscles, including increased pelvic floor tone, instability of the pelvic floor muscles, poor pelvic floor muscle control, and phobic avoidance. Initial goals of physical therapy are to increase the patient's awareness of her pelvic floor muscles, increase her ability to locate, contract, and fully relax these muscles, and to address reflex guarding and/or muscle spasm (vaginismus reflex).

The gynecologist can help women by teaching them three simple physical maneuvers: a Kegel's maneuver, a reverse Kegel's maneuver, and superficial perineal massage. The rationale for the exercises should be explained to the woman, ie, to assess her control and awareness of these muscles. During the pelvic examination, the clinician can ask the woman to locate and contract the pubococcygeus muscles. Instruct the patient to contract around the examination finger; she can use a quick contraction ( 3 seconds) and release ( 3 seconds) and then a long contraction ( 10 seconds) and release (10 seconds). The gynecologist should provide feedback as to whether or not she is using the correct pelvic floor muscles or other accessory muscles (abdomen, leg, and buttocks) to help with the contraction. Patients should be encouraged to practice contracting and fully relaxing the pelvic floor muscles regularly throughout the day (10 sets of contraction followed by relaxation three times per day). This should increase her awareness about how to control and relax her pelvic floor muscles. Patients can also be instructed on how to perform a reverse Kegel's maneuver and/or Valsalva maneuver. This maneuver can help relax or "push out" the superficial pelvic floor muscles, thereby increasing the capacity of the introitus. Patients find this a useful strategy to employ prior to inserting anything into the vagina.

Superficial perineal massage is often taught to pregnant woman in the hope of avoiding perineal trauma during delivery. Likewise, it can be used to help the woman with vulvodynia to gradually desensitize the area through touching and to increase the pliability of her superficial pelvic floor muscles. Instruct the woman to use a lubricant and/or massage oil to insert her thumb into her vagina (one inch) and then press down towards the anus until the stretch is uncomfortable but not painful. She should then hold that position for one minute while focusing on relaxing the pelvic floor, then slowly massage the lower vagina using a U-shaped movement from side to side for up to 3 minutes a day. The woman's partner can be instructed on how to massage the muscles using their index finger, and can do this prior to any penetrative activity.

Many women with sexual pain demonstrate a guarding reflex in anticipation of pain. While this may be evident during clinical examination, it may be a result of anticipation 
of pain and/or secondary to a painful examination, and not necessarily reflective of what happens in the sexual setting. Patients with severe pelvic floor muscle tightness and phobia will describe feeling like there is something "blocking" the vagina, and the partner has to push past this obstacle to penetrate. The couple may not be able to consummate their relationship. If severe anxiety is present, then this involuntary contraction of the superficial pelvic floor muscles will result in a diminished vaginal opening and increased friction and/ or pressure when anything is inserted into the vagina. Many women will benefit greatly by treating their anxiety around penetration by using vaginal inserts in a stepwise manner, from small insert to large insert, using inserts by themselves and then in the presence of a partner, and using inserts in a nonsexual and then sexual setting. Again the gynecologist can provide guidance on where to purchase the inserts and how to use them. Work with a mental health professional is often key to overcoming the associated anxiety and avoidance.

While some women may be able to address their pelvic floor muscle control once given clear guidance, many will need the expert care of a pelvic floor physiotherapist. Pelvic floor physiotherapists offer a range of therapies including: biofeedback to reduce resting pelvic floor muscle tone; pelvic floor exercises to identify, isolate, and improve control of the pelvic floor muscles; and myofascial release, trigger point pressure, and massage therapy.

\section{Medical therapy}

In our multidisciplinary vulvodynia program, women (primarily with PVD) begin treatment by adhering to the above recommendations regarding symptom management, psychosexual counseling, and pelvic floor physiotherapy. The program comprises 12 appointments over 12 weeks. At the time of discharge, the vast majority of women will notice an improvement in symptoms and are motivated to pursue the above therapies. ${ }^{45}$ However, there is a small proportion of women who wish to pursue other medical and/or surgical therapies.

A variety of medical therapies have been proposed by experts for the treatment of vulvodynia (Table 5). Despite numerous publications reporting over 30 different therapeutic interventions, research regarding the medical treatment of vulvodynia has failed to identify a convincing therapeutic intervention. ${ }^{46-48}$ Given the hypothesis that the chronic discomfort may be secondary to central and peripheral nervous system sensitization, oral pain adjuvants (eg, antidepressants and anticonvulsants) are often recommended as first-line treatment for vulvodynia. The quality of research in this area
Table 5 Examples of medical therapies applied to vulvodynia

\begin{tabular}{lll}
\hline Medication applied to & PVD & GVD \\
\hline Betamethasone/lidocaine injections & $\times$ & \\
Botulinum toxin injections & $\times$ & $\times$ \\
Interferon injections & $\times$ & \\
Oral calcium citrate & $\times$ & \\
Oral TCA antidepressants (amitriptyline, desipramine) & $\times$ & $\times$ \\
Oral SSRI antidepressants (duloxetine) & & $\times$ \\
Oral antifungals (fluconazole, ketoconazole) & $\times$ & \\
Oral anticonvulsants (gabapentin, pregabalin) & $\times$ & $\times$ \\
Topical 5\% lidocaine & $\times$ & $\times$ \\
Topical capsaicin & $\times$ & \\
Topical cromolyn & $\times$ & \\
Topical gabapentin & $\times$ & $\times$ \\
Topical montelukast & $\times$ & \\
Topical nifedipine & $\times$ & \\
Topical nitroglycerin & $\times$ & \\
\hline
\end{tabular}

Abbreviations: PVD, provoked vestibulodynia; GVD, generalized vulvodynia.

is variable, with the majority of studies being descriptive rather than experimental. Three recent systematic and critical reviews of this literature have failed to find sufficient evidence to support the recommendation of using oral pain adjuvants to treat vulvodynia. ${ }^{49-51}$ The ideal vulvodynia patient, type of oral pain adjuvant medication, route of medication delivery, and dose and duration of therapy are still unknown to treat vulvodynia when prescribing either antidepressants or anticonvulsants. Descriptive studies regarding use of antidepressants, such as tricyclic antidepressants and serotonin reuptake inhibitors, to treat vulvodynia report success rates of $27 \%-100 \%{ }^{49}$ However, a well-designed randomized controlled trial by Foster et al failed to find a therapeutic effect of tricyclic antidepressants with or without $5 \%$ lidocaine versus placebo. ${ }^{52}$ The majority of published studies used tricyclic antidepressants, ie, amitriptyline, desipramine, or nortriptyline. Doses reported ranged from $10 \mathrm{mg}$ to $225 \mathrm{mg}$ daily. Most women need to be started at a low dose and slowly titrated over weeks to a higher dose. The side effects may limit the dose that can be sustained. Side effects associated with tricyclic antidepressants include sedation, constipation, dry mouth, cognitive dysfunction, and sexual dysfunction. The duration of treatment reported in the studies ranged from 4 weeks to 30 months. ${ }^{49}$

The studies reviewed regarding the use of anticonvulsants, eg, gabapentin, pregabalin, or lamotrigine, to treat vulvodynia have reported success rates in the range of $50 \%-82 \% .^{50,51}$ The majority of these descriptive studies used oral gabapentin starting at a dose of $300 \mathrm{mg}$ per day, increasing by $300 \mathrm{mg}$ per week to a maximum of 1,200 mg per day. Doses up to $3,000 \mathrm{mg}$ are reported. ${ }^{50}$ Anticonvulsants are reported to be better tolerated than antidepressants, with 
fewer side effects. Side effects associated with gabapentin include sedation, sleep disturbances, nausea, vomiting, dizziness, and headache. This medication has an effect on sexual function, and gabapentin-induced anorgasmia has been reported in women. ${ }^{53}$ The impact of this medication on sexual function in women with vulvar pain is unknown because the studies did not include sexual function as an outcome. The reported duration of treatment ranged from 4 weeks to 30 months. A multicenter, randomized clinical trial is currently underway to examine the effectiveness of extended-release gabapentin in women with sexual pain secondary to provoked vestibulodynia. ${ }^{54}$

Based on my clinical experience, I believe there is a legitimate role for oral pain adjuvants in treating a select group of women with vulvodynia. I use oral pain adjuvants as a first-line therapy only for women with moderate to severe daily vulvar pain that is interfering with their quality of life and/or activities of daily living. These women are often motivated to tolerate medication side effects, and remain on the medication for the sustained period of time needed to notice improvements over 3-6 months. I believe oral pain adjuvants have limited if any benefit in treating women who are primarily experiencing sexual pain. Patient adherence is critical for success. Start at a low test dose for several days, gradually titrate up the dose to the recommended dose over a month as tolerated, and set realistic time expectations. Side effects are a common complaint and should be managed aggressively. A woman may need to try several different medications before finding one that she can tolerate. All patients should be advised not to abruptly discontinue medication but rather to taper off slowly.

\section{Surgical therapy}

There are case reports of success using nerve stimulation via implanted electrodes ${ }^{55}$ and serial local and regional nerve blocks ${ }^{56}$ to treat chronic daily vulvar pain. Experimental trials and long-term follow-up are needed to see if either procedure is effective. In regards to the surgical treatment of sexual pain secondary to provoked vestibulodynia, the literature would suggest that surgery is an effective intervention. ${ }^{46-48}$ Reported success rates range from $61 \%$ to $94 \%{ }^{48}$ One of the most common procedures, the modified vestibulectomy, involves excision of the hymenal ring from the 3 to 9 o'clock position and of the superficial vestibular mucosa to Hart's line. There are many methodologic problems with the studies, and questions still remain regarding: for whom the treatment is effective (prognostic patient characteristics); what specific surgical procedures are best (perineoplasty, modified vestibulectomy, focal excision); and the long-term outcomes in regards to pain and sexual function. Many patients and clinicians would consider surgical intervention as a last resort. In a randomized controlled trial by Bergeron et al comparing biofeedback, cognitive behavioral therapy, and vestibulectomy, all participating women reported improvement over time. In fact, at the 2.5 year follow-up, the women who received cognitive behavioral therapy reported a reduction in sexual pain similar to that in the group of women who had the surgical intervention (modified vestibulectomy). ${ }^{57}$

A comprehensive analysis of the research regarding medical and surgical treatments for vulvodynia is beyond the scope of this paper, and interested readers are directed to the excellent published critical reviews in this area. ${ }^{46-48}$ While the lack of evidence for treatment effectiveness should not limit clinical practice, in general I believe that clinicians should avoid recommending medical treatments that are based on case reports from small numbers of patients and are potentially harmful to patients (eg, capsaicin cream, botulinum toxin A, vaginal diazepam). Many of the medical therapies proposed (eg, cromolyn cream, nifedipine, botulinum injections, fluconazole) in small case series have ultimately not been found to be superior to placebo in treating vulvodynia in well designed studies. ${ }^{58-61}$

\section{Placebo effect}

Finally, the placebo intervention effect $(>50 \%$ decrease in pain) seen in the treatment of vulvodynia may account for up to $33 \%$ of reported improvement. ${ }^{46}$ Clinicians should consider this when interpreting "success" rates of any given intervention. Giving the women's pain a "name", validating the pain as real, and then giving her a treatment for which she can expect pain relief can all have a powerful influence on her perception and subsequent control over the pain, highlighting the importance of thoughts, emotions, and feelings. The improvement in both treatment and placebo groups in the randomized controlled trials in this area may also indicate a high background remission rate for vulvodynia. ${ }^{58-61}$ In a recent study, Davis et $\mathrm{al}^{62}$ reported that $41 \%$ of women with sexual pain secondary to vulvodynia who did not participate in any treatment reported a significant improvement of their pain over a 2-year interval. Women who reported trying any type of treatment (physical, psychological, sexual, surgical, and/or medical) all reported significant reductions in pain and psychosexual improvement over this period of time. There was no difference between the treatments in diminishing pain. Thus the gynecologist can be optimistic when counseling women with vulvodynia, that they can expect to see an improvement in their pain with treatment. 


\section{Conclusion}

Vulvodynia is a chronic pain condition that is best understood in terms of a combination of biological, psychological, and social factors affecting a woman's health, ie, the biopsychosocial model of illness. The management of women with vulvodynia should be individualized, holistic, and if possible multidisciplinary. The gynecologist has a key role in the assessment of not only a woman's vulvar sensitivity, but also her pelvic floor muscle health, her thoughts and reactions to her pain, and her sexual health. The important initial steps in management include excluding other causes for her pain, providing education about chronic pain and the impact of pain on health, and providing recommendations for symptom management. The gynecologist may focus subsequent visits on assessing further and addressing the woman's pelvic floor muscles and function and psychosexual health. Referral of women with vulvodynia to other relevant health care providers (pelvic floor physiotherapists, sexual health counselors, cognitive behavioral therapists) is strongly recommended to not only address pain but also the negative impact of chronic pain on overall quality of life.

\section{Disclosure}

The author reports no conflict of interest in this work.

\section{References}

1. Haefner HK. Report of the International Society for the Study of Vulvovaginal Disease: terminology and classification of vulvodynia. J Low Genit Tract Dis. 2007;11:48-49.

2. Edwards L. Subsets of vulvodynia: overlapping characteristics. J Reprod Med. 2004;49:883-887.

3. Clare CA, Yeh J. Vulvodynia in adolescence: childhood vulvar pain syndromes. J Pediatr Adolesc Gynecol. 2011;24:110-115.

4. Reed BD, Harlow SD, Sen A, et al. Prevalence and demographic characteristics of vulvodynia in a population-based sample. Am J Obstet Gynecol. 2012;206:170. e1-e9.

5. Moyal-Barracco M, Labat JJ. Vulvodynia and chronic pelvic and perineal pain. Prog Urol. 2010;20:1019-1026.

6. Xie Y, Shi L, Xiong X, Wu E, Veasley C, Dade C. Economic burden and quality of life of vulvodynia in the United States. Curr Med Res Opin. 2012;28:601-608.

7. Sadownik LA. Clinical profile of vulvodynia patients: a prospective study of 300 patients. J Reprod Med. 2000;45:679-684.

8. Nunns D, Mandal D. Psychological and psychosexual aspects of vulvar vestibulitis. Genitourin Med. 1997;73:541-544.

9. Arnold L, Bachmann GA, Rosen R, Kelly S, Rhoads GG. Vulvodynia: characteristics and associations with comorbidities and quality of life. Obstet Gynecol. 2006;107:617-624.

10. Ehrstrom S, Kornfeld D, Rylander E, Bohm-Starke N. Chronic stress in women with localised provoked vulvodynia. J Psychosom Obstet Gynaecol. 2009;30:73-79.

11. Schover LR, Youngs DD, Cannata R. Psychosexual aspects of the evaluation and management of vulvar vestibulitis. Am J Obstet Gynecol. 1992;167:630-636.

12. Gates EA, Galask RP. Psychological and sexual functioning in women with vulvar vestibulitis. J Psychosom Obstet Gynaecol. 2001;22:221-228.
13. Bergeron S, Binik YM, Khalifé S, et al. A randomized comparison of group cognitive-behavioral therapy, surface electromyographic biofeedback, and vestibulectomy in the treatment of dyspareunia resulting from vulvar vestibulitis. Pain. 2001;91:297-306.

14. Hinchliff S, Gott M, Wylie K. A qualitative study of heterosexual women's attempts to renegotiate sexual relationships in the context of severe sexual problems. Arch Sex Behav. 2012;41:1253-1261.

15. Rosen NO, Bergeron S, Glowacka M, Delisle I, Baxter ML. Harmful or helpful: perceived solicitous and facilitative partner responses are differentially associated with pain and sexual satisfaction in women with provoked vestibulodynia. J Sex Med. 2012;9:2351-2360.

16. Connor JJ, Robinson B, Wieling E. Vulvar pain: a phenomenological study of couples in search of effective diagnosis and treatment. Fam Process. 2008;47:139-155.

17. Ventolini G. Vulvar pain: anatomic and recent pathophysiologic considerations. Clin Anat. 2013;26:130-133.

18. Babula O, Bongiovanni AM, Ledger WJ, Witkin SS. Immunoglobulin E antibodies to seminal fluid in women with vulvar vestibulitis syndrome: relation to onset and timing of symptoms. Am J Obstet Gynecol. 2004;190:663-667.

19. Sarma AV, Foxman B, Bayirli B, Haefner H, Sobel JD. Epidemiology of vulvar vestibulitis syndrome: an exploratory case-control study. Sex Transm Infect. 1999;75:320-326.

20. Ventolini G, Gygax SE, Adelson ME, Cool DR. Vulvodynia and fungal association: a preliminary report. Med Hypotheses. 2013;81:228-230.

21. Nguyen RH, Swanson D, Harlow BL. Urogenital infections in relation to the occurrence of vulvodynia. J Reprod Med. 2009;54: 385-392.

22. Bornstein J, Goldschmid N, Sabo E. Hyperinnervation and mast cell activation may be used as histopathologic diagnostic criteria for vulvar vestibulitis. Gynecol Obstet Invest. 2004;58:171-178.

23. Chadha S, Gianotten WL, Drogendijk AC, et al. Histopathologic features of vulvar vestibulitis. Int J Gynecol Pathol. 1998;17:7-11.

24. Kahn BS, Tatro C, Parsons CL, Willems JJ. Prevalence of interstitial cystitis in vulvodynia patients detected by bladder potassium sensitivity. J Sex Med. 2010;7:996-1002.

25. Reed BD, Payne CM, Harlow SD, Legocki LJ, Haefner HK, Sen A. Urogenital symptoms and pain history as precursors of vulvodynia: a longitudinal study. J Womens Health (Larchmt). 2012;21:1139-1143.

26. Hampson JP, Reed BD, Clauw DJ, et al. Augmented central pain processing in vulvodynia. J Pain. 2013;14:579-589.

27. Pukall CF, Binik YM, Khalifé S, Amsel R, Abbott FV. Vestibular tactile and pain thresholds in women with vulvar vestibulitis syndrome. Pain. 2002;96:163-175.

28. Foster DC, Dworkin RH, Wood RW. Effects of intradermal foot and forearm capsaicin injections in normal and vulvodynia-afflicted women. Pain. 2005;117:128-136.

29. Giesecke J, Reed BD, Haefner HK, Giesecke T, Clauw DJ, Gracely RH. Quantitative sensory testing in vulvodynia patients and increased peripheral pressure pain sensitivity. Obstet Gynecol. 2004;104: 126-133.

30. Leeuw M, Goossens ME, Linton SJ, Crombez G, Boersma K, Vlaeyen JW. The fear-avoidance model of musculoskeletal pain: current state of scientific evidence. J Behav Med. 2007;30:77-94.

31. Payne KA, Binik YM, Pukall CF, Thaler L, Amsel R, Khalife S. Effects of sexual arousal on genital and non-genital sensation: a comparison of women with vulvar vestibulitis syndrome and healthy controls. Arch Sex Behav. 2007;36:289-300.

32. Basson R. The recurrent pain and sexual sequelae of provoked vestibulodynia: a perpetuating cycle. J Sex Med. 2012;9:2077-2092.

33. Khandker M, Brady SS, Vitonis AF, MacLehose RF, Stewart EG, Harlow BL. The influence of depression and anxiety on risk of adult onset vulvodynia. J Women's Health (Larchmt). 2011;20:1145-1451.

34. Brotto LA, Basson R, Gehring D. Psychological profiles among women with vulvar vestibulitis syndrome: a chart review. J Psychosom Obstet Gynaecol. 2003;24:195-203. 
35. Jantos M, Burns NR. Vulvodynia. Development of a psychosexual profile. J Reprod Med. 2007;52:63-71.

36. Lynch PJ. Vulvodynia as a somatoform disorder. J Reprod Med. 2008;53:390-396.

37. Nguyen RH, Ecklund AM, Maclehose RF, Veasley C, Harlow BL. Co-morbid pain conditions and feelings of invalidation and isolation among women with vulvodynia. Psychol Health Med. 2012;17: 589-598.

38. Hartmann D. Chronic vulvar pain from a physical therapy perspective. Dermatol Ther. 2010;23:505-513.

39. Davies S, Quintner J, Parsons R, et al. Preclinic group education sessions reduce waiting times and costs at public pain medicine units. Pain Med. 2011;12:59-71.

40. Tan O, Bradshaw K, Carr BR. Management of vulvovaginal atrophyrelated sexual dysfunction in postmenopausal women: an up-to-date review. Menopause. 2012;19:109-117.

41. Goetsch MF. Unprovoked vestibular burning in late estrogendeprived menopause: a case series. J Low Genit Tract Dis. 2012;16: 442-446.

42. Reed BD, Haefner HK, Sen A, Gorenflo DW. Vulvodynia incidence and remission rates among adult women: a 2-year follow-up study. Obstet Gynecol. 2008;112:231-237.

43. Masheb RM, Kerns RD, Lozano C, Minkin MJ, Richman S. A randomized clinical trial for women with vulvodynia: cognitivebehavioral therapy vs supportive psychotherapy. Pain. 2009;141 $31-40$.

44. Brotto LA, Sadownik L, Thomson S. Impact of educational seminars on women with provoked vestibulodynia. J Obstet Gynaecol Can. 2010;32:132-138.

45. Sadownik LA, Seal BN, Brotto LA. Provoked vestibulodynia - women's experience of participating in a multidisciplinary vulvodynia program. J Sex Med. 2012;9:1086-1093.

46. Andrews JC. Vulvodynia interventions - systematic review and evidence grading. Obstet Gynecol Surv. 2011;66:299-315.

47. Bergeron S, Binik YM, Khalifé S, Pagidas K. Vulvar vestibulitis syndrome: a critical review. Clin J Pain. 1997;13:27-42.

48. Landry T, Bergeron S, Dupuis MJ, Desrochers G. The treatment of provoked vestibulodynia: a critical review. Clin J Pain. 2008;24:155-171.

49. Leo RJ, Dewani S. A systematic review of the utility of antidepressant pharmacotherapy in the treatment of vulvodynia pain. $J$ Sex Med. 2013;10:2497-2505.

50. Leo RJ. A systematic review of the utility of anticonvulsant pharmacotherapy in the treatment of vulvodynia pain. J Sex Med. 2013;10:2000-2008.
51. Spoelstra SK, Borg C, Weijmar Schultz WC. Anticonvulsant pharmacotherapy for generalized and localized vulvodynia: a critical review of the literature. J Psychosom Obstet Gynaecol. 2013;34: $133-138$.

52. Foster DC, Kotok MB, Huang LS, et al. Oral desipramine and topical lidocaine for vulvodynia: a randomized controlled trial. Obstet Gynecol. 2010;116:583-593.

53. Drabkin R, Calhoun L. Anorgasmia and withdrawal syndrome in a woman taking gabapentin. Can J Psychiatry. 2003;48: 125-126.

54. Brown CS, Foster DC, Wan JY, Rawlinson LA, Bachmann GA; Gabapentin (GABA) Study Group. Rationale and design of a multicenter randomized clinical trial of extended release gabapentin in provoked vestibulodynia and biological correlates of response. Contemp Clin Trials. 2013;36:154-165.

55. De Andres J, Sanchis-Lopez N, Asensio-Samper JM, Fabregat-Cid G, Dolz VM. Peripheral subcutaneous vulvar stimulation in the management of severe and refractory vulvodynia. Obstet Gynecol. 2013;121: 495-498.

56. McDonald JS, Rapkin AJ. Multilevel local anesthetic nerve blockade for the treatment of generalized vulvodynia: a pilot study. J Sex Med. 2012;9:2919-2926.

57. Bergeron S, Khalifé S, Glazer HI, Binik YM. Surgical and behavioral treatments for vestibulodynia: two-and-one-half year follow-up and predictors of outcome. Obstet Gynecol. 2008;111:159-166.

58. Nyirjesy P, Sobel JD, Weitz MV, Leaman DJ, Small MJ, Gelone SP. Cromolyn cream for recalcitrant idiopathic vulvar vestibulitis: results of a placebo controlled study. Sex Transm Infect. 2001;77:53-57.

59. Bornstein J, Tuma R, Farajun Y, Azran A, Zarfati D. Topical nifedipine for the treatment of localized provoked vulvodynia: a placebo-controlled study. J Pain. 2010;11:1403-1409.

60. Petersen CD, Giraldi A, Lundvall L, Kristensen E. Botulinum toxin type A-a novel treatment for provoked vestibulodynia? Results from a randomized, placebo controlled, double blinded study. J Sex Med. 2009;6:2523-2537.

61. Bornstein J, Livnat G, Stolar Z, Abramovici H. Pure versus complicated vulvar vestibulitis: a randomized trial of fluconazole treatment. Gynecol Obstet Invest. 2000;50:194-197.

62. Davis SN, Bergeron S, Binik YM, Lambert B. Women with provoked vestibulodynia experience clinically significant reductions in pain regardless of treatment: results from a 2-year follow-up study. J Sex Med. 2013;10:3080-3087.
International Journal of Women's Health

\section{Publish your work in this journal}

The International Journal of Women's Health is an international, peerreviewed open-access journal publishing original research, reports, editorials, reviews and commentaries on all aspects of women's healthcare including gynecology, obstetrics, and breast cancer. The manuscript management system is completely online and includes

\section{Dovepress}

a very quick and fair peer-review system, which is all easy to use. Visit http://www.dovepress.com/testimonials.php to read real quotes from published authors. 